\title{
Magnetic excitations of interface pinned domains
}

\author{
Ana L. Dantas ${ }^{\mathrm{a}}$, A.S. Carriço ${ }^{\mathrm{b}, *}$ \\ ${ }^{a}$ Departamento de Física, Universidade do Estado do Rio Grande do Norte, Mossoró-RN 59610-210, Brazil \\ ${ }^{\mathrm{b}}$ Departamento de Física, Centro de Ciências Exatas, Campus Universitário, Universidade Federal do Rio Grande do Norte, \\ Natal-RN 59078-970, Brazil
}

Received 25 August 2000; received in revised form 24 January 2001

\begin{abstract}
We study the uniform excitations of the domains of a thin magnetic film on a two sublattice antiferromagnetic substrate. An interface step defect stabilizes a domain wall which holds two large ferromagnetic domains with opposite magnetization on each side of the defect line. The orientation of the magnetization in the domains, in response to an external field, parallel to the film surface, differs from the single domain situation and leads to new dispersion relations for the uniform precession modes of the ferromagnetic film. We apply the model to the case of a Nèel wall of a uniaxial ferromagnetic film and discuss the implications of the results in measurements of the interface effective exchange field. (C) 2001 Published by Elsevier Science B.V.
\end{abstract}

PACS: $75.70 . \mathrm{Cn} ;$ 75.70.Ak; 75.60.Ch

Keywords: F/AF interfaces; Exchange bias; Step defects; Uniaxial films; FMR

\section{Introduction}

The magnetic properties of thin film multilayers are to a large extent influenced by the nature of the interfaces. In nanometer size samples a considerable fraction of the atoms belong in the interface regions. In recent years a great deal of research effort has been dedicated to the study of the nature of the interfaces between an antiferromagnetic (AF) substrate and a thin ferromagnetic (F) film. $\mathrm{F} / \mathrm{AF}$ bilayers are commonly used, as part of magnetic sensor devices, such as spin-valves exhibiting giant magneto-resistance, whenever a

\footnotetext{
*Corresponding author. Fax: + 55-84-2153-791.

E-mail address: acarrico@dfte.ufrn.br (A.S. Carriço).
}

reference ferromagnetic layer is needed. In these structures the AF substrate is used to stabilize large domains in the ferromagnetic film. A large volume of results has been produced so far for the characterization of $\mathrm{F} / \mathrm{AF}$ interfaces [1]. However, there are still unsolved issues and the microscopic nature of the $\mathrm{F} / \mathrm{AF}$ interface is currently a subject of discussion.

The interface biasing observed in $\mathrm{F} / \mathrm{AF}$ bilayers, when cooled from the Nèel temperature of the AF layer in the presence of an external magnetic field, is known for a few decades [2]. In the simplest model of the interface effects, as proposed originally to explain the interface biasing in $\mathrm{Co}$ fine particles embedded in the parent oxide [2], the substrate effect is represented by an exchange field $\left(H_{\mathrm{E}}\right) . H_{\mathrm{E}}$ is attributed to a Heisenberg coupling of 
the magnetic moments at the interface, and should be equal to the hysteresis biasing. However, the observed values of the hysteresis shift are two to three orders of magnitude smaller than what might be expected if $H_{\mathrm{E}}$ is of the order of the intrinsic exchange field of the ferromagnetic film [3]. The formation of domain walls, either running along the interface or perpendicular to it, has been evoked in order to explain the order of magnitude of the measured hysteresis shifts of field cooled F/AF bilayers [3,4].

Magnetization measurements sample macroscopic areas of the interface and averages out the impact of microscopic structure of the interface on the ferromagnetic thin film. Thus, in a rough interface, where both AF sublattices may appear at the interface, the net effect on the ferromagnetic film may be strongly reduced, in comparison to the local value of the interface exchange field.

It was recently shown [5] that narrow domain walls may form in the ferromagnetic film, in response to interface roughness. The frequency of the rigid displacement excitations of these domain walls depends on the value of the interface coupling in interface areas of microscopic size and fall in the range of the uniform excitations of the domains for a wide range of values of the interface field $\left(H_{\mathrm{A}}<H_{\mathrm{E}}<40 H_{\mathrm{A}}\right)[6]$.

While the measurement of the excitations of interface pinned domain walls may bring valuable information about the local value of the interface exchange field and the degree of interface roughness [6], the measurement of the uniform excitations of the domains that may form in rough $\mathrm{F} / \mathrm{AF}$ bilayers is also of interest. The existence of an interface step defect produces a pair of domains of opposite magnetization which are indirectly coupled via the interface.

The existence of domains subjected to opposite interface fields, resulting from areas of the interface containing opposite sublattices of the AF substrate, produces relevant modifications in the field dependence of the uniform mode excitations. In a ferromagnetic resonance (FMR) measurement one may find new results.

In this paper, we study the excitations of long wavelength of a pair of domains, separated by a domain wall which is pinned to an interface step defect. We consider an uniaxial thin ferromagnetic film and the external field parallel and perpendicular to the interface step defect. We find that the modifications in the frequencies of the uniform modes are controlled by the relative value of the interface and anisotropy fields $\left(H_{\mathrm{E}} / H_{\mathrm{A}}\right)$. In this paper we use $H_{\mathrm{J}}$ to denote the interface exchange field $\left(H_{\mathrm{E}}\right)$.

In the Section 2, we discuss the model used to describe the system and calculate the excitations for external field applied parallel to the step edge. Section 3 is devoted to the study of the excitations when the external field is perpendicular to the step edge. Our conclusions are presented in the Section 4.

\section{External field applied along the step edge}

We calculate the excitations of two domains separated by a Nèel wall pinned at a step defect in a F/AF interface. The system consists of a thin ferromagnetic film, with in-plane magnetization, on a two-sublattice uniaxial antiferromagnetic substrate as shown in Fig. 1. The anisotropy axis of the antiferromagnet is parallel to the easy direction of the ferromagnet (the $z$-axis). The substrate step edge runs along the $z$-axis and divides the interface in two regions, each one containing spins from a sublattice of the antiferromagnet. In our model no relaxation is allowed for

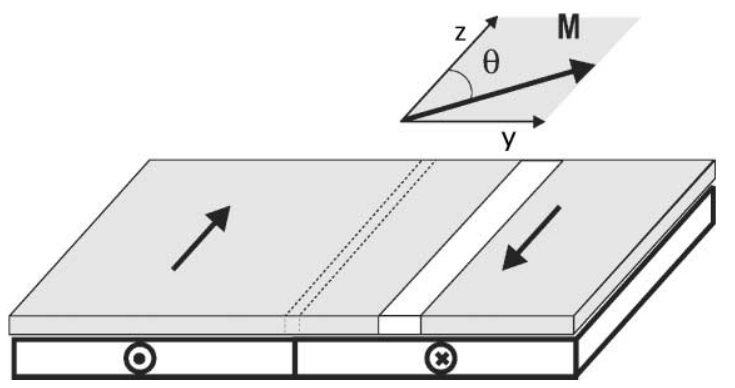

Fig. 1. Schematic representation of a Nèel wall pinned at a step defect on an antiferromagnetic substrate. The domain wall is displaced from the pinning center by the external field. The dashed line narrow stripe, in the center of the figure, indicates the position of the domain wall, located at the step defect, for $H=0$. 
the substrate spins, which are held fixed along the anisotropy direction.

We do not consider any variation of the magnetization along the $z$-axis or $x$-axis directions. The nucleation of a Nèel wall in the ferromagnetic film follows from the discontinuous change of direction of the interface exchange field at the step edge.

The magnetic energy per unit wall area is given by

$$
\begin{aligned}
E= & \int_{-L}^{L}\left[A\left(\frac{\partial \theta}{\partial y}\right)^{2}-(H M+J(y)) \cos \theta\right. \\
& \left.-K \cos ^{2} \theta\right] \mathrm{d} y
\end{aligned}
$$

where

$$
J(y)=\left\{\begin{array}{cc}
J, & y<0, \\
-J, & y>0 .
\end{array}\right.
$$

$L$ is the width of the AF domains on each side of the step defect and $M$ is the saturation value of the magnetization. The first term in the Eq. (1) is the intrinsic exchange energy density, the second term is the Zeeman energy density for an external field of strength $H$ applied along the direction $\hat{z}$, the third term is the interface coupling energy density and the last term is the uniaxial anisotropy energy density. Along the text we make reference to the interface exchange field, given by $H_{\mathrm{J}}=J / M$, and to the anisotropy field $H_{\mathrm{A}}=2 K / M$. Also, we use $H_{\mathrm{A}}=0.55 \mathrm{kOe}$ and $4 \pi M=18 \mathrm{kOe}$, as appropriate to $\mathrm{Fe}$.

Our previous results indicate that the domain wall is stable, holding two opposite domains, if the external field strength is smaller than the value of the effective interface exchange field $\left(H_{\mathrm{J}}\right)$. This result was originally predicted on the basis of a numerical simulation of the equilibrium structure of the ferromagnetic film [5] and confirmed by variational calculations [6]. For large values of the external field strength the displacement of the domain wall center from the step defect increases exponentially with the strength of the external field and for $H=H_{\mathrm{J}}$ the domain wall detaches from the interface step defect [6].

The existence of opposite domains may be of interest in the interpretation of FMR measure-

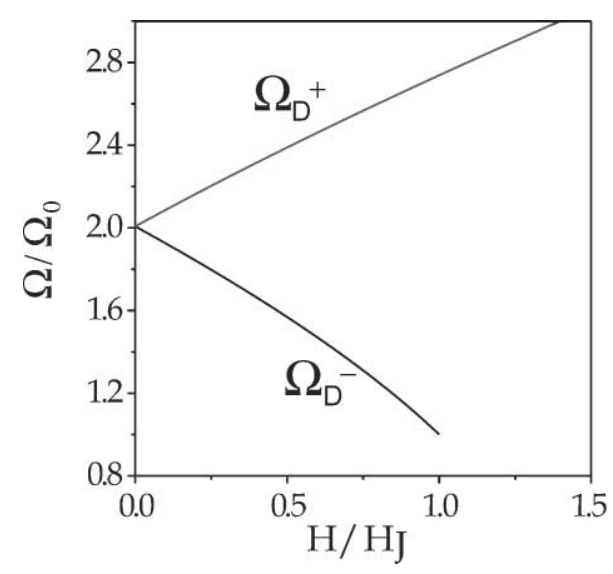

Fig. 2. Frequency of the uniform modes of the domains for $H_{\mathrm{J}}=1.5 \mathrm{kOe}$. The frequencies are shown in units of $\Omega_{0}=$ $\sqrt{H_{\mathrm{A}}\left(4 \pi M+H_{\mathrm{A}}\right)}$ and the external field is shown in units of the interface field strength $H_{\mathrm{J}}$.

ments. The interface exchange field may actually be larger than the fields used in resonance experiments. In this case one might be able to observe two kinds of domain modes: the domain with magnetization opposite to the external field and the domain with magnetization aligned with the external field.

In Fig. 2 we show the frequencies of the uniform mode of the domains for an interface exchange field of $H_{\mathrm{J}}=1.5 \mathrm{kOe}$. The qualitative features seen for this value of $H_{\mathrm{J}}$ have also been seen for other values of $H_{\mathrm{J}}$.

One of the domains is subjected to an effective field of $H_{\mathrm{J}}-H$, while the other domain is subjected to an effective field of $H_{\mathrm{J}}+H$. Thus, the frequency of one of the domain modes decreases while the frequency of the other domain mode increases as the external field increases.

The frequencies of the domain modes are given by [7]:

$$
\Omega_{\mathrm{D}}^{-}=\gamma \sqrt{\left(H_{\mathrm{J}}-H+H_{\mathrm{A}}\right)\left(H_{\mathrm{J}}-H+H_{\mathrm{A}}+4 \pi M\right)}
$$

and

$$
\Omega_{\mathrm{D}}^{+}=\gamma \sqrt{\left(H_{\mathrm{J}}+H+H_{\mathrm{A}}\right)\left(H_{\mathrm{J}}+H+H_{\mathrm{A}}+4 \pi M\right)} .
$$

Note that for $H<H_{\mathrm{J}}$, the domain wall is pinned 


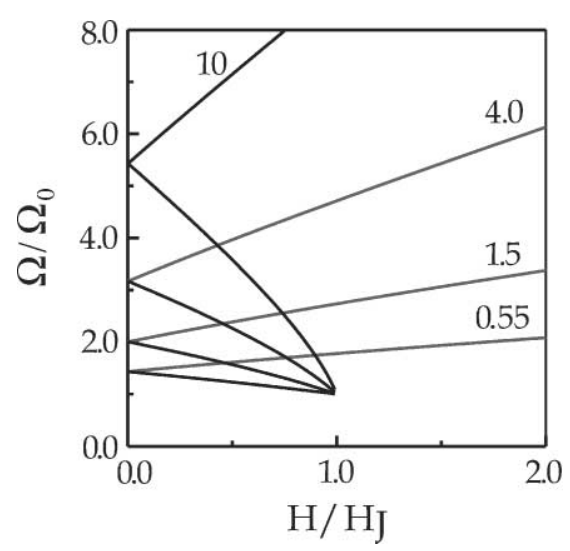

Fig. 3. Frequency of the uniform modes of the domains. The frequencies are shown in units of $\Omega_{0}$ and the external field in shown in units of the interface field strength $H_{\mathrm{J}}$. The number by the curves indicate the strength of the interface exchange field $H_{\mathrm{J}}$ in kOe.

at the step defect and there are two domain modes. When $H>H_{\mathrm{J}}$ the domain wall is liberated from the step defect. Then the magnetization is saturated in the direction of the applied field and just one mode survives.

The dispersion of the uniform modes of the interface pinned domains correspond to two lines starting at $\Omega_{\mathrm{D}}^{+}=\Omega_{\mathrm{D}}^{-}=$ $\gamma \sqrt{\left(H_{\mathrm{J}}+H_{\mathrm{A}}\right)\left(H_{\mathrm{J}}+H_{\mathrm{A}}+4 \pi M\right)}$, for $H=0$. The difference between the frequencies, for a given value of the external field, increases when $H_{\mathrm{J}}$ increases. These features are shown in Fig. 3 where we display the domain frequencies for a few values of the interface exchange field. It is clear that the curves corresponding to $\Omega_{\mathrm{D}}^{-}$converge to the same value for $H=H_{\mathrm{J}}$. This is predicted in Eq. (3). When the external field strength approaches the interface exchange field, $H \cong H_{\mathrm{J}}$, the net field is nearly zero on the domain with magnetization opposite to the external field. Thus, for any value of $H_{\mathrm{J}}, \Omega_{\mathrm{D}}^{-}\left(H_{\mathrm{J}}\right)=\Omega_{0}$.

\section{External field applied perpendicular to the step edge}

When the external field is applied perpendicular to the step edge, along the $y$-axis direction, the domain wall does not move. The magnetization at

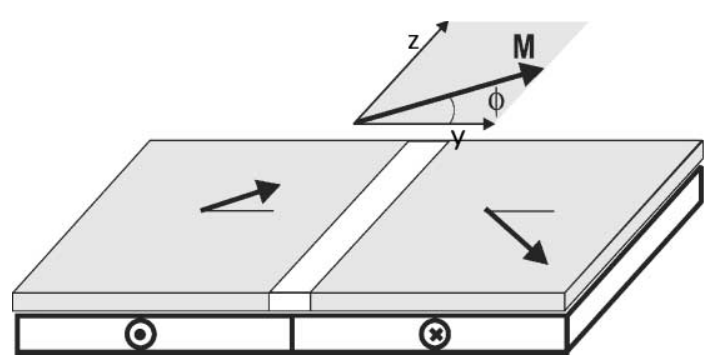

Fig. 4. Schematic representation of a Nèel wall pinned at a step defect on an antiferromagnetic substrate, under the effect of a transverse external field. The magnetization at the domains turns in the field direction and the domain wall center stays pinned at the step defect at $y=0$.

the domain wall center is parallel to the external field and the leading external field effects consist in modifying the domain wall width and also turning the magnetization in the domains in the direction perpendicular to the step edge. Our previous results [5] indicate that if external field strength is small compared to the intrinsic exchange field of the ferromagnetic film the modifications in the domain wall width are rather small.

The magnetic energy is given by:

$$
\begin{aligned}
E= & \int_{-L}^{L}\left[A\left(\frac{\partial \phi}{\partial y}\right)^{2}-(H M \cos \phi\right. \\
& \left.+J(y) \sin \phi)-K \sin ^{2} \phi\right] \mathrm{d} y,
\end{aligned}
$$

where $\phi$ is the angle between the magnetization and the external field, as indicated in Fig. 4.

The value of the angles $\left( \pm \phi_{0}\right)$ at the domains are obtained by minimizing the energy density in the domains. We have:

$h \sin \phi_{0}-j \cos \phi_{0}-\sin \phi_{0} \cos \phi_{0}=0$,

where, $h=H M / 2 K$ and $j=J / 2 K$.

$\phi_{0}$ is obtained from the numerical solution of Eq. (6). In Fig. 5 we show $\phi_{0}(H)$ for a few values of the interface exchange field strength. It is readily seen that the effect of the interface field is to make $\phi_{0}(H) \neq 0$ for any value of $H$. Thus complete saturation of magnetization is only achieved for a very large value of the external field.

Although the external field is perpendicular to the easy axis, the effective field acting in each 


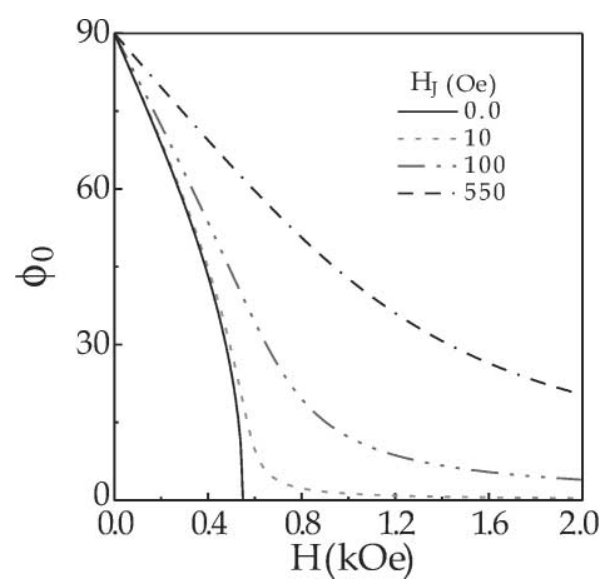

Fig. 5. Domain angle as a function of the transverse field strength.

domain, $\boldsymbol{H}_{\mathrm{ef}}=\boldsymbol{H}+\boldsymbol{H}_{\mathrm{J}}$, changes both in direction and strength when the external field is increased.

Only for extremely large values of the external field the effective field is nearly perpendicular to the uniaxial axis. Thus the complete alignment of the magnetization in the domains with the external field, when the external field reaches the anisotropy field value, is not attained. This is the reason why the domain excitation frequency is affected by the step defect.

From Fig. 5 it is readily seen that for $H_{\mathrm{J}}=0$ the domains are aligned with the external field, for $H=H_{\mathrm{A}}$. However, if $H_{\mathrm{J}} \neq 0$ the magnetization at the domains does not align with the external field. For large values of $H_{\mathrm{J}}$ the angle at the domains decrease very slowly, when the external field is increased.

This field dependence of $\phi_{0}$ controls the field dependence of the frequency of the domain modes.

The frequency of uniform excitations of the domains depend on the angle $\phi_{0}$ between $\boldsymbol{M}$ and $\boldsymbol{H}$ in the domains. The external field is perpendicular to the uniaxial axis and for any value of $H$ both domains exhibit the same excitation frequency. The long wave length domain mode frequency is given by [7]

$$
\begin{aligned}
& \Omega_{\mathrm{D}}= \\
& \quad \gamma \sqrt{\left(\frac{H}{\cos \phi_{0}}+4 \pi M\right)\left(H \cos \phi_{0}-H_{\mathrm{A}} \cos 2 \phi_{0}+H_{\mathrm{J}} \sin \phi_{0}\right)} .
\end{aligned}
$$

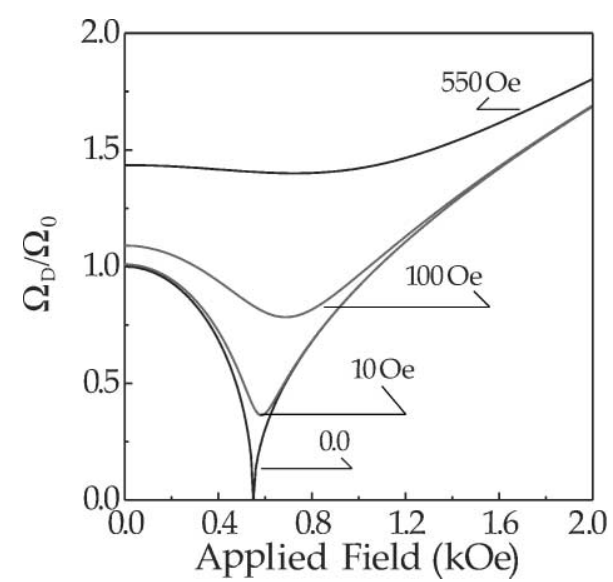

Fig. 6. Frequency of the uniform mode of the domains in units of $\Omega_{0}$. The numbers by the curves indicate the values of the interface exchange field.

Note that in the limit of small values of the external field strength $(H)$, we get from Eq. (6):

$\frac{H}{\cos \phi_{0}}=H_{\mathrm{A}}+H_{\mathrm{J}}$.

Thus, the limit of Eq. (7) for small values of the external field strength $(H)$ is

$\Omega_{\mathrm{D}}=\gamma \sqrt{\left(H_{\mathrm{J}}+H_{\mathrm{A}}+4 \pi M\right)\left(H_{\mathrm{J}}+H_{\mathrm{A}}\right)}$,

which reproduces the values obtained in Eqs. (3) and (4).

As seen in Fig. 6 the field dependence of the uniform domain mode is strongly affected by the value of interface exchange field. If $H_{\mathrm{J}}=0$ we obtain the dispersion of a free uniaxial ferromagnetic film. The frequency goes to zero when the external field strength is equal to the anisotropy field. For modest values of $H_{\mathrm{J}}$ the dispersion is similar to that of a free uniaxial film, but if $H_{\mathrm{J}}$ is comparable or larger than $H_{\mathrm{A}}$ the dispersion is strongly modified. For values of $H_{\mathrm{J}}$ comparable to the value of $H_{\mathrm{A}}$ the dispersion is extremely flat, for small values of the external field, as indicated in the Fig. 6 for $H_{\mathrm{J}}=550 \mathrm{Oe}$. This field dependence of $\Omega_{\mathrm{D}}$ is in clear contrast with that expected in the absence the interface effects. 


\section{Final remarks}

In this paper the spectrum of the uniform mode of oscillations of a pair of domains of a thin uniaxial ferromagnetic film on an AF substrate was discussed. In the absence of external field the pair of domains, with opposite magnetizations, is stabilized by the interface exchange field, which changes the direction in a step defect running along the uniaxial axis of the ferromagnet.

The domain excitations were studied as a function of the external field strength. Two orientations of the external field with respect to the uniaxial axis of the ferromagnet were considered. Our results are summarized below.

(i) If the external field is applied perpendicular to the domains, both domains oscillate with the same frequency.

We have shown that the pair of domains may respond to the external field in a rather different way, if compared to a single domain sample with $H_{\mathrm{J}}=0$. Only in the limit of rather weak values of the interface exchange field, compared to the anisotropy of the ferromagnetic film, will the results look like what one might expect for a single domain.

For small values of the interface exchange field, the frequency of the domain oscillations drops to nearly zero when $H=H_{\mathrm{A}}$ and is an increasing function of $H$ for $H>H_{\mathrm{A}}$. However, as seen in Fig. 6, if the interface exchange field is comparable to, or larger than the anisotropy field, the frequency of the domain oscillations is a rather flat function of the external field, if $H$ is not too large.

(ii) If the external field is applied along the uniaxial anisotropy direction there are two modes: the domains on each side of the step defect oscillate with different frequencies $\Omega \frac{ \pm}{\mathrm{D}}(H)$, for $H \neq 0$.

For $H=0$ the frequencies are: $\Omega_{\mathrm{D}}^{ \pm}=$ $\gamma \sqrt{\left(H_{\mathrm{J}}+H_{\mathrm{A}}\right)\left(H_{\mathrm{J}}+H_{\mathrm{A}}+4 \pi M\right)}$. Note that for strong values of the interface exchange field, $\left(H_{\mathrm{J}} \gg H_{\mathrm{A}}\right), \Omega_{\mathrm{D}}^{ \pm}(0)$ is nearly proportional to $H_{\mathrm{J}}$.

The frequency of the domain with magnetization along the external field direction is an increasing function of the external field strength. However, the frequency of the domain with the magnetization, pinned by the interface field opposite to the external field, is a decreasing function of the external field strength.

The mode with frequency $\Omega_{\mathrm{D}}^{-}$, is not commonly expected to be observed in the FMR of a single film and might help in the identification of $\mathrm{F} / \mathrm{AF}$ interface roughness. For any value of $H_{\mathrm{J}}$, the frequency of this mode has a minimum value of $\Omega_{0}$, for $H=H_{\mathrm{J}}$.

For a given value of the external field $\left(H<H_{\mathrm{J}}\right)$ the difference between the frequencies of the domains is large if the interface exchange field is large, as predicted by Eq. (3) and (4). This is shown in Fig. 3 for a few values of the interface exchange field.

For $H>H_{\mathrm{J}}$ the ferromagnetic film is saturated in the direction of the applied field, and just the mode with frequency $\Omega_{\mathrm{D}}^{+}(H)$ may be excited. This is the commonly observed single domain mode, apart from the effect of the interface field $H_{\mathrm{J}}$ which is added to the external field $H$.

Experiments with $\mathrm{Fe} / \mathrm{Cr} / \mathrm{Fe}$ trilayers, where the $\mathrm{Cr}$ spacers were in the form of a wedge, revealed a short period coupling [8]. The observed periodic structure of domains in the thin $\mathrm{Fe}$ films were found to be in good correspondence with the terraces formed in the $\mathrm{Cr}$ surface. Step defects separate the terraces and the interface exchange field changes direction at the step defects inducing the nucleation of narrow domain walls in the Fe film [8].

Similar studies of $\mathrm{Fe} / \mathrm{Cr}$ vicinal bilayers have been conducted more recently. By changing the miscut angle the surface density of step defects was modified and a reorientation of the magnetization of the Fe film has been observed. For low values of the miscut angle multidomain structures were observed, particularly for thin Fe films [9].

Our results might be helpful to study the domain excitations in these vicinal systems [8,9], where a periodic domain structure exists, when the miscut angle is small, so that the domains are much larger than the domain wall width.

One interesting situation arises when the step density is large so that one may wonder if a one to one correspondence exists between each interface step defect and an interface pinned domain wall. 
The answer to this question involves the knowledge of the strength of the interface exchange coupling $\left(H_{\mathrm{J}}\right)$. At first sight one might expect that if the average step distance is comparable to the intrinsic domain wall width of the ferromagnet, then the interface step defect would not nucleate a domain wall. This is true provided there is no interface effect on the domain wall width itself. However, it has been shown [5,6] that the domain wall width may be strongly reduced if the interface exchange field strength is large. This was shown to be particularly important for low anisotropy ferromagnets.

Thus in order to study the relation between the step density and the density of interface pinned domain walls one needs to know the value of the interface exchange field. This interesting aspect of the problem is beyond the scope of the present work. However, if the interface step density is moderately large but a fraction of the step defects pinn domain walls, then our results apply. In this case the peculiar form of the dispersion of the uniform modes of the domains, exhibited in Figs. 2 and 6 might help in the interpretation of resonance experiments on $\mathrm{F} / \mathrm{AF}$ bilayers. Furthermore, it might help to estimate the strength of the interface exchange field.

\section{Acknowledgements}

The authors thank the Brazilian Agencies CNPq and CAPES for partial financial support.

\section{References}

[1] D.T. Pierce, J. Unguris, R.J. Celotta, M.D. Stiles, J. Magn. Magn. Mater. 200 (1999) 290.

[2] W.H. Meiklejohn, C.P. Bean, Phys. Rev. 105 (1957) 904.

[3] A.P. Malozemoff, Phys. Rev. B 35 (1987) 3679.

[4] D. Mauri, H.C. Siegmann, P.S. Bagus, E. Kay, J. Appl. Phys. 62 (1987) 3047.

[5] A.L. Dantas, A.S. Carriço, J. Phys.: Condens. Matter 11 (1999) 2707.

[6] A.L. Dantas, A.S. Carriço, R.L. Stamps, Phys. Rev. B 62 (2000) 8650.

[7] T.E. Hasty, L J. Boudreaux, J. Appl. Phys. 32 (1961) 1807.

[8] J. Unguris, R.J. Celotta, D.T. Pierce, Phys. Rev. Lett. 67 (1991) 140.

[9] E.J. Escorcia-Aparicio, J.H. Wolfe, H.J. Choi, W.L. ling, R.K. Kawakami, Z.Q. Qiu, Phys. Rev. B 59 (1999) 11892. 